\title{
A case of superior segmental optic hypoplasia accompanied by a glaucomatous optic neuropathy
}

\author{
Ikuyo Ohguro \\ Hiroshi Ohguro \\ Department of Ophthalmology, \\ Sapporo Medical University School \\ of Medicine, Sapporo, Hokkaido, Japan
}

\begin{abstract}
This is the first case report of a bilateral superior segmental optic hypoplasia (SSOH) accompanied by a glaucomatous optic neuropathy (GON). A 47-year-old man incidentally diagnosed as having bilateral SSOH, simultaneously disclosed glaucomatous optic disc appearances, including enlargements of the cup of the optic nerve heads and a thinning of the infero-temporal neuroretinal rim with laminar dot sign accompanied by a retinal nerve fiber layer (RNFL) local defect of infero-temporal region in the right eye. The visual field examination revealed that the corresponding nasal step, arcuate scotoma and RNFL field defects in the right eye.
\end{abstract}

Keyword: retinal nerve fiber layer

\section{Introduction}

Superior segmental optic hypoplasia ( $\mathrm{SSOH}$ ), a congenital anomaly of the optic nerve head and the retina, is clinically characterized by four ophthalmoscopic findings of disc and retina. Namely, these are: superior entrance of the central retinal artery, superior peripapillary scleral halo, pallor of the superior disc, and thinning of the superior peripapillary retinal nerve fiber layer (RNFL) (Petersen and Walton 1977; Bjork et al 1978; Nelson et al 1986; Kim et al 1989; Brodsky et al 1993; Landau et al 1998; Purvin 2002; Foroozan 2005). The patients with SSOH usually have relatively good visual acuities with specific visual field changes of inferior altitudinal or sector defect connecting to the blind spot, and often are misdiagnosed as glaucomatous optic neuropathy (GON). GON, a progressive disease of the optic nerve head and the retina, is ophthalmoscopically characterized by enlargement of the cup of the optic nerve head, thinning of the neuroretinal rim predominantly in the infero-temporal or superotemporal region accompanied by an association with decreased RNFL thickness, and visual field defects such as paracentral or arcuate scotoma, nasal step, and RNFL field defects (Shields 1997).

Optical coherence tomography (OCT), a new diagnostic tool that can perform tomography/cross-sectional imaging of optic nerve head and retina, demonstrated supero-nasal defect of the neuroretinal rim of the optic nerve head associated with decreased RNFL thickness in patients with $\mathrm{SSOH}$, whereas infero-/supero-temporal defect was disclosed in patients with GON (Unoki et al 2002; Gupta et al 2006). Thus, in view of the localized thinning of the neuroretinal rim of the optic nerve head, the regional difference between supero-nasal in SSOH and infero-/supero-temporal in GON is the clinically important point to differentiate SSOH from GON.

Although optic disc changes are very similar in $\mathrm{SSOH}$ and GON, as described above, their disease etiology and clinical course are significantly different. In fact, as far as we know, no combined case of $\mathrm{SSOH}$ and GON has been reported prior to this interesting case presenting with bilateral $\mathrm{SSOH}$ in conjunction with glaucoma-like RNFL field defects. 


\section{Case report}

A 47-year-old man complaining of deterioration of bilateral near and far visions over a couple of months was referred to our clinic in September 2006. He had been medicated for essential hypertension over a period of 15 years. His mother had suffered from glaucoma.

On our ophthalmic examination, his best corrected visual acuity was $1.2 \times-5.5 \mathrm{D}$ OD, and $1.2 \times-6.5 \mathrm{D}$ OS. No abnormalities were detected in pupil reaction, color vision, eye position, ocular movement, anterior segment of the eye, or ocular media. His intraocular pressures (IOPs) were 12 $\mathrm{mmHg}$ in both eyes. The gonioscopic examination revealed normal wide open angle with slight pigmentation in both eyes. No abnormal findings were detected in total blood cell count or serum blood chemistry examinations.

As shown in Figure 1, the fundus examination revealed that the cup-to-disc (C/D) ratio was 0.83 in the right eye and 0.7 in the left, associated with the following characteristics in bilateral optic discs: 1) oblique insertion of oval shaped optic nerve heads tilted to the temporal side with temporal peripapillary atrophy, 2) superior entrance of the central retinal arteries, and 3 ) thinning of supero-nasal neuroretinal rims with double ring sign accompanied by diffuse defect of the superior peripapillary RNFL. In addition, thinning of infero-temporal neuroretinal rim with infero-temporal RNFL wedge defect and laminar dot sign due to exposure of fenestrate in lamina cribrosa were simultaneously associated to the right optic disc.

A vertical OCT scan through the optic nerve head rotated 30 degrees nasal showed a defect of the supero-nasal portion of the optic disc with thinned RNFL in the both eyes, especially in the left eye and thinning of RNFL in the infero-temporal region as well as the supero-nasal region in the both eyes, especially in the right eye (Figure 2). For quantitative assessment of RNFL, a circular OCT scan at $3.4 \mathrm{~mm}$ diameter through the center of the optic disc demonstrated that the average of RNFL thickness of all quadrants except temporal ones was abnormally thin in both eyes and that the RNFL thickness of the infero-temporal region was further decreased in the right eye (Figure 3).

A static perimetry (Humphrey field analyzer program 30-2) showed an arcuate scotoma with nasal step in the supero-nasal field in the right eye, and a dense sector defect connecting to the blind spot in the infero-temporal field in the left (Figure 4). A kinetic perimetry (Goldmann perimeter) also showed a number of depressions of the peripheral isoptors in the infero-/supero-temporal fields in the right eye (Figure 4).

After first examinations, his IOPs have been lower than $21 \mathrm{mmHg}$ and no abnormalities have been found in head computed tomography images.

\section{Discussion}

In 1989, Kim and colleagues (1989) reviewed 10 patients with $\mathrm{SSOH}$, all of whom were the children of diabetic mothers, and described 4 characteristic findings in the optic disc: 1) relative superior entrance of the central retinal artery, 2) pallor of the superior disc, 3) superior peripapillary halo, and 4) thinning of the superior peripapillary RNFL. However recently Yamamoto and colleagues (2004) suggested that a superior peripapillary scleral halo and pallor of the superior disc are not always present since only one eye in 9 eyes of $12 \mathrm{SSOH}$ patients showed all the four characteristics whereas the rest of these eyes lacked a superior scleral halo and superior disc pale (Hashimoto et al 1999; Unoki et al 2002). Therefore, they advocated that the clinical definition of SSOH should be thinning of the neuroretinal rim of the optic nerve head most prominent in the superior nasal region with corresponding RNFL defects in the superior nasal region in at least one eye.

In the present case, two of the above four characteristic disc findings were observed, namely, relatively superior entrance of the central retinal artery and thinning of the superior peripapillary RNFL thickness. In addition, the depressions of the peripheral isoptors in the infero-temporal fields in the right eye and a dense sector defect connecting to the blind spot in the infero-temporal field in the left eye were disclosed by perimetry. Thus, he has diagnosed as having bilateral $\mathrm{SSOH}$, even though his mother did not have diabetes mellitus. In addition, our case also presented glaucomatous optic disc appearances such as 1) enlargements of the cup of the optic nerve heads resulting in 0.83 and 0.7 of $\mathrm{C} / \mathrm{D}$ ratios in right eye and left eye, respectively, and 2) a thinning of the infero-temporal neuroretinal rim with laminar dot sign accompanied by an RNFL local defect in the infero-temporal region, corresponding nasal step, arcuate scotoma and RNFL field defects in the right eye. The average RNFL thickness of the inferior quadrant as well as the superior was abnormally thin in both eyes, especially in the right eye. However, his IOPs did not exceed $21 \mathrm{mmHg}$ and there were no alternative causes of glaucoma-like visual field defects. Therefore taken together with these findings, he was diagnosed as normal tension glaucoma in addition to $\mathrm{SSOH}$. Since it is known that $40 \%$ axonal loss is required for there to be any change detectable by visual field analysis (Quigley et al 1980), the recognition of RNFL loss in patients with normal visual fields, has led us to the concept of "pre-perimetric" glaucoma, signifying early glaucomatous damage not evident on standard automated perimetry. Accordingly, our present case was thought to be advanced glaucoma stage in his right eye and 


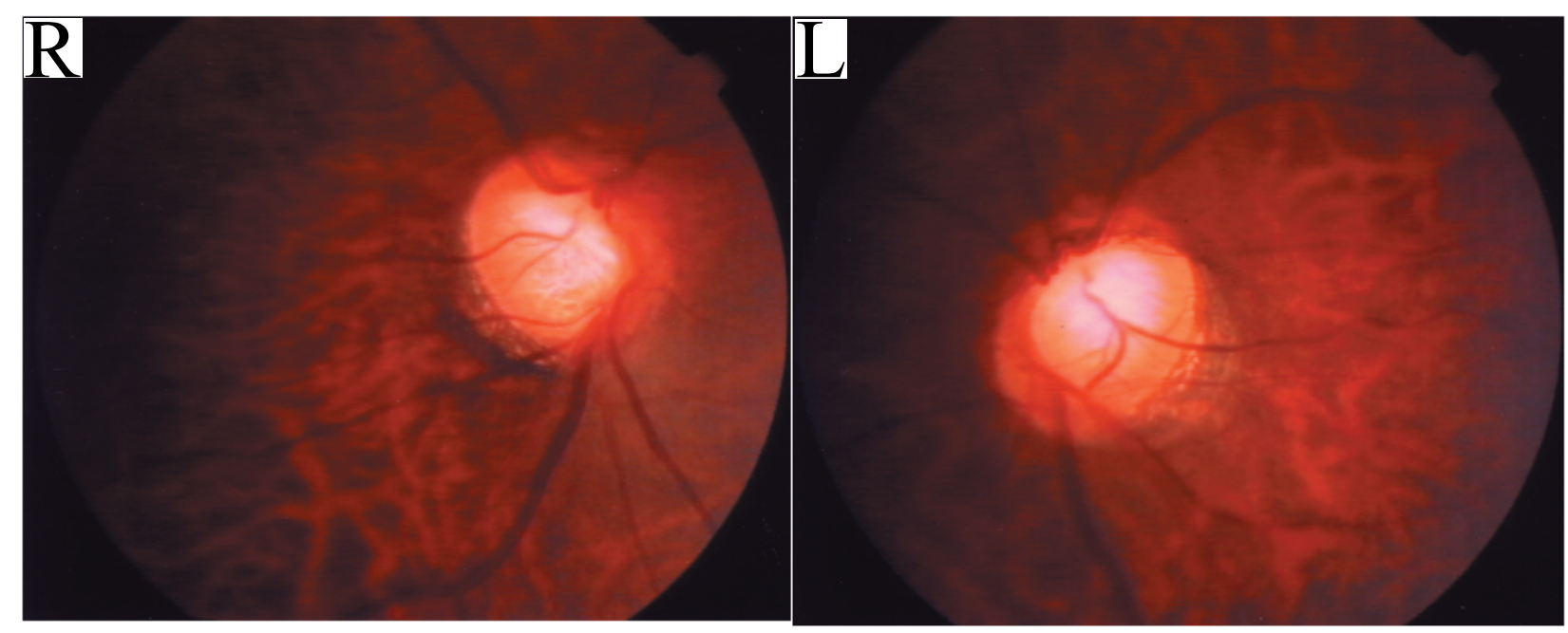

Figure I Fundus photographs of the patient. Fundus photographs showing: I) oblique insertion of oval shaped optic nerve heads tilted to temporal side with temporal peripapillary atrophy, 2) superior entrance of the central retinal arteries, and 3) thinning of supero-nasal neuroretinal rims with double ring sign accompanied by diffuse defect of the superior peripapillary RNFL in both eyes and, simultaneously, thinning of infero-temporal neuroretinal rim with infero-temporal RNFL wedge defect and laminar dot sign due to exposure of fenestrate in lamina cribrosa in the right eye.

Abbreviation: RNFL, retinal nerve fiber layer.
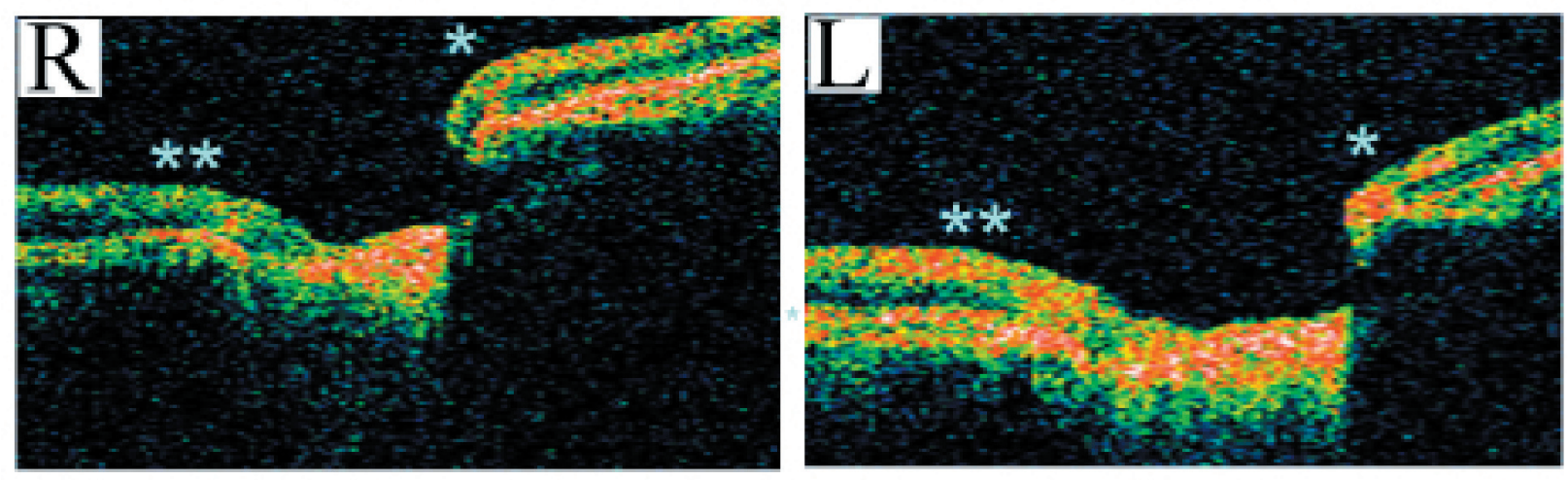

Figure 2 Vertical OCT scans through the optic nerve head rotated 30 degrees nasal in the patient.Vertical OCT scans through the optic nerve head rotated 30 degrees nasal depicting defect of the supero-nasal portion of the optic disc in the both eyes, especially in the left eye (indicated asterisk) and thinning of RNFL in the infero-temporal region as well as the supero-nasal region in the both eyes, especially in the right eye (indicated double asterisk).

Abbreviation: OCT, optical coherence tomography.
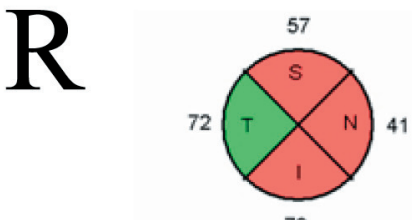

79

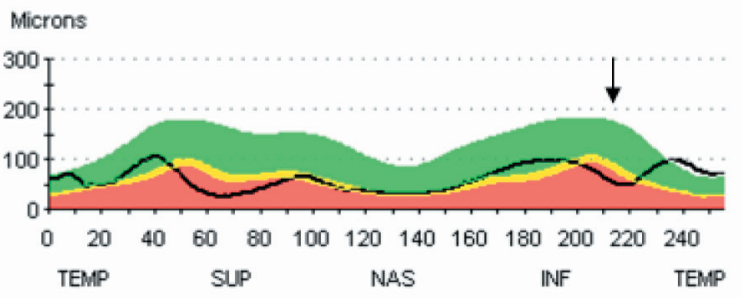

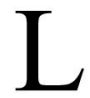

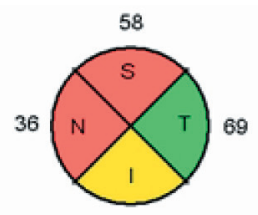

94

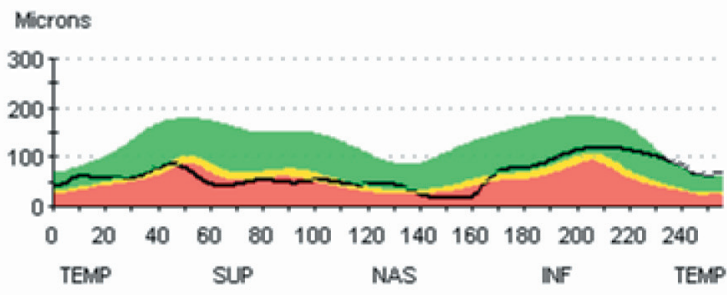

Figure 3 Circular OCT scans at $3.4 \mathrm{~mm}$ diameter through the center of the optic disc. Circular OCT scans at $3.4 \mathrm{~mm}$ diameter through the center of the optic disc demonstrating abnormal thinning of average RNFL thickness of all quadrants except temporal in both eyes and further decreased RNFL thickness of infero-temporal region in the right eye (indicated arrow).

Abbreviations: OCT, optical coherence tomography; RNFL, retinal nerve fiber layer. 


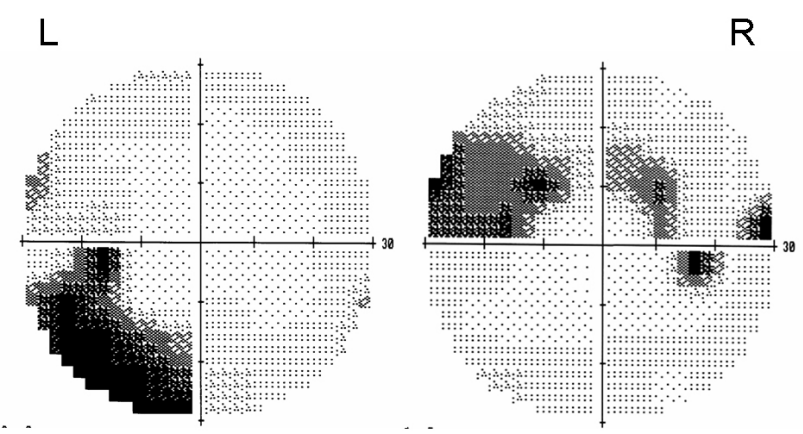

Figure $4 \mathrm{~A}$ static perimetry in the patient. A static perimetry showing an arcuate scotoma with nasal step in the supero-nasal field in the right eye, and a dense sector defect connecting to the blind spot in the infero-temporal field in the left eye.

pre-perimetric glaucoma stage in left. However, our search of the literature disclosed that there have been no cases of $\mathrm{SSOH}$ accompanied by glaucoma so far.

Although the etiology of SSOH has not been clarified yet, it was suggested that maternal diabetes is most likely to be involved since SSOH frequently occurs in offspring of type 1 diabetic mothers (Nelson et al 1986; Kim et al 1989; Brodsky et al 1993; Landau et al 1998; Foroozan 2005). In fact, Unoki and colleagues (2002) reported familial cases of SSOH who were offspring of type 1 diabetic mothers, and suggested the involvement of a genetic factor. Diabetes mellitus is also known to be a risk factor for the development and progression of GON (Richler et al 1982; Zeiter et al 1991) since diabetes is a disease of small vessels, and compromise of the microcirculation of the optic disc is a possible contributing mechanism in the pathogenesis of GON. However, Hashimoto and colleagues (1999) reported 4 Japanese patients with SSOH whose mothers were not diabetic and our case did not have diabetes mellitus or a diabetic mother, suggesting that some unknown mechanisms could disturb the normal development of retina and optic nerve during the fatal period in patients with SSOH. If such events do happen in $\mathrm{SSOH}$, we can speculate that impaired retinal development during the fetal period of maternal diabetes and other mechanisms may cause vulnerable optic disc and retina and will likely develop to GON.

\section{References}

Bjork A, Laurell CG, Laurell U. 1978. Bilateral optic nerve hypoplasia with normal visual acuity. Am J Ophthalmol, 86:524-9.

Brodsky MC, Schroeder GT, Ford R. 1993. Superior segmental optic hypoplasia in identical twins. J Clin Neuro-Ophthalmol, 13:152-4.

Foroozan R. 2005. Superior segmental optic nerve hypoplasia and diabetes mellitus. J Diabetes Complications, 19:165-7.

Gupta V, Gupta A, Dogra MR. 2006. Principles of OCT scanning in glaucoma. In: Atlas optical coherence tomography of macular diseases and glaucoma, 2nd ed. New Delhi, India, Jaypee Brothers Med Pub.

Hashimoto M, Ohtsuka K, Nakagawa T, et al. 1999. Topless optic disk syndrome without maternal diabetes mellitus. Am J Ophthalmol, 128:111-12.

Kim RY, Hoyt WF, Lessel S, et al. 1989. Superior segmental optic hypoplasia. A sign of maternal diabetes mellitus. Am J Ophthalmol, 107:1312-15.

Landau K, Bajka JD, Kirchschlager BM. 1998. Topless optic disks in children of mohters with type I diabetes mellitus. Am J Ophthalmol, 125:605-11.

Nelson M, Lessell S, Sadun AA. 1986. Optic nerve hypoplasia and maternal diabetes mellitus. Arch Neurol, 43:20-5.

Petersen RA, Walton DS. 1977. Optic nerve hypoplasia with good visual acuity and visual field defects. Arch Ophthalmol, 95:254-8.

Purvin VA. 2002. Superior segmental optic nerve hypoplasia. J Neuroophthalmol, 22:116-17.

Quigley HA, Miller NR, George T. 1980. Clinical evaluation of nerve fiber layer atrophy as an indicator of glaucomatous optic nerve damage. Arch Ophthalmol, 98:1564-71.

Richler M, Werner EB, Thomas D. 1982. Risk factors for progression of visual field defects in medically treated patients with glaucoma. Can J Ophthalmol, 17:245-8.

Shields MB. 1997. Optic nerve head and peripapillary retina. In: Textbook of glaucoma, 4th ed. Maryland, USA, Williams and Wilkins.

Unoki K, Ohba N, Hoyt WF. 2002. Optical coherence tomography of superior segmental optic hypoplasia. Br J Ophthalmol, 86:910-14.

Yamamoto T, Sato M, Iwase A. 2004. Superior segmental optic hypoplasia found in Tajimi Eye Health Care Project participants. Jpn J Ophthalmol, 48:578-83.

Zeiter JH, Shin DH, Baek NH. 1991. Visual field defects in diabetic patients with primary open-angle glaucoma. Am J Ophthalmol, 111:581-4. 